\title{
PAPER
}

\section{Measurement and analysis of vibration in the myocardium telescopic motion for novel echo-graphic diagnosis}

\author{
Hiroshi Kanai $^{1}$, Shin-ichi Katsumata ${ }^{1}$, Hideyuki Honda ${ }^{2}$ and Yoshiro Koiwa ${ }^{3}$ \\ ${ }^{1}$ Graduate School of Engineering, Tohoku University, \\ Aramaki-aza-Aoba 05, Sendai, 980-8579 Japan \\ ${ }^{2}$ Faculty of Medical Science and Welfare, Tohoku Bunka Gakuen University, \\ Kunimi 6-45-16, Aoba-ku, Sendai, 981-8551 Japan \\ ${ }^{3}$ Graduate School of Medicine, Tohoku University, \\ Seiryou-machi 1-1, Aoba-ku, Sendai, 980-8574 Japan
}

(Received 21 December 2001, Accepted for publication 10 July 2002)

\begin{abstract}
We have already developed a novel ultrasonic-based method, namely, the phased tracking method, to measure the rapid velocity in the heart wall by accurately tracking the movement in the heart wall. By applying this method to in vivo experiments, the velocity signal of the heart wall with small amplitude, less than several micrometers on the large motion resulting from a heartbeat, can be successfully detected. There are high frequency components of up to several hundreds Hertz, which have not been previously investigated at all. In this study, after the papillary muscle had been extracted from an isolated rat heart, it was electrically stimulated, and the resultant muscular vibration was directly measured in the frequency range up to $2 \mathrm{kHz}$ using a laser Doppler velocimeter in order to confirm if the high frequency vibrations occur during contraction and relaxation. For the injured myocardium, the power increase in the high frequency components becomes smaller. The experimental results support the hypothesis that the high frequency components are included in the velocity signal measured for the first time in the human interventricular septum by the phase tracking method using ultrasound.
\end{abstract}

Keywords: Phased tracking method, Myocardium vibration, Myocardium vulnerability

PACS number: 43.80.Qf, 43.80.Vj

\section{INTRODUCTION}

We have already developed a novel ultrasonic-based method, namely, the phased tracking method, to measure the rapid velocity in the heart wall by accurately tracking the movement $x_{i}(t)$ of a point $(i)$ in the heart wall by the constraint least-squares method applied to both the phase and magnitude of the quadrature-demodulated signals [1]. RF pulses are transmitted from an ultrasonic transducer at a pulse repetition interval $\Delta T$, and the reflected ultrasonic wave is received by the same transducer and is multiplied by the original RF signal in quadrature-demodulation. By synchronizing the pulse transmission timing and the acquisition timing in $\mathrm{A} / \mathrm{D}$ conversion rigidly with the original RF signal, the achieved lower limit $\left|v_{\min }\right|$ in the above velocity measurement was $0.1 \mathrm{~mm} / \mathrm{s}$, which was confirmed in phantom experiments [2], using the developed method and system [1]. The lower limit depends on the small dispersion in the above synchronization and on the additive noise in the received signal. Since the lower limit $\left|v_{\min }\right|$ is less than $1 / 2,000$ of the upper limit $\left|v_{\max }\right|$ of the measurable velocity, which is free from aliasing, the dynamic range of the velocity measurement is given by $20 \log _{10}\left|v_{\max }\right| /\left|v_{\min }\right|=73 \mathrm{~dB}$. In other phantom experiments [1], a vibration with small amplitude of $20 \mu \mathrm{m}$ generated on the large motion with an amplitude of $\pm 7.5 \mathrm{~mm}$ and a frequency of $1 \mathrm{~Hz}$, which simulates the heartbeat, was successfully measured in the range from $1 \mathrm{~Hz}$ to $1 \mathrm{kHz}$.

By applying this method and system to in vivo experiments, the velocity signal, $v\left(t ; x_{i}\right)$, of point $i$ at a depth of $x_{i}$ in the heart wall with small amplitude, less than several micrometers on the large motion resulting from a heartbeat, can be successfully detected with reproducibility [1]. By applying frequency analysis to the resultant velocity signal, $v\left(t ; x_{i}\right)$, of the heart wall, the Fourier spectrum of $v\left(t ; x_{i}\right)$ has been obtained for the first time with sufficient reproducibility in the frequency range up to 
$100 \mathrm{~Hz}$ [1]. The developed method has already been realized in a real-time measurement system [3]. Moreover, from the difference of the resultant velocity signals $\left\{v\left(t ; x_{i}\right)\right\}$ between point $x_{i}$ and its succeeding point $x_{i+1}$ preset along the ultrasonic beam, a minute change in thickness of several tens of micrometers has been detected with a spatial resolution of $0.5 \mu \mathrm{m}$ [4] as described above.

With the phased tracking method it is possible to accurately detect small amplitude velocity signals of less than a few micrometers in the heart wall [1]. There are high frequency components up to several hundreds Hertz [1] which cannot be recognized in standard B-mode echocardiography, M-mode echocardiography, or in conventional tissue Doppler imaging (TDI). The clinical and physiological meanings and the mechanisms of such high frequency vibrations have not been previously investigated. If the mechanism of the high frequency components can be clarified, it will offer potential for novel quantitative assessment of myocardial viability.

It is known that vibrations along the muscular fibers occur during contraction and relaxation of the skeletal muscle [5-9]. In the literature, there have been many reports of attempts to transcutaneously measure such muscle sounds originating from the muscle vibrations [5-9]. However, the muscle vibrations have been measured only in the frequency range up to $100 \mathrm{~Hz}$.

In this paper, after the papillary muscle was extracted from the isolated rat heart, it was electrically stimulated during perfusion with physiological solution, and the resultant muscle vibration was directly measured in the frequency range up to $2 \mathrm{kHz}$ using a laser Doppler velocimeter. The detected vibrations were then analyzed in the frequency domain in order to confirm that high frequency vibrations occur during contraction and relaxation of the heart muscle.

\section{IN VITRO EXPERIMENTS}

A papillary muscle extracted from a rat heart was fixed in a water tank by two tungsten hooks and a force transducer was employed to measure the generated tension $T(t)$ along the longitudinal axis of the myocardium as shown in Fig. 1. In order to maintain the physiological conditions close to in vivo conditions, Krebs-Henseleit solution was circulated in the water tank by the peristaltic pump and the temperature of the water tank was kept constant at $25 \pm 0.2$ degrees Celsius.

The myocardium was electrically stimulated through two parallel platinum electrodes by rectangular wave $e(t)$ with a duration of $1 \mathrm{~ms}$ and a repetition frequency of $1 \mathrm{~Hz}$ as shown in Fig. 3(a). At the same time, the velocity component $v(t)$ generated in the direction normal to that of the myocardium was measured at the center of the muscle by a laser Doppler velocimeter (Ono Sokki LV-1300) as

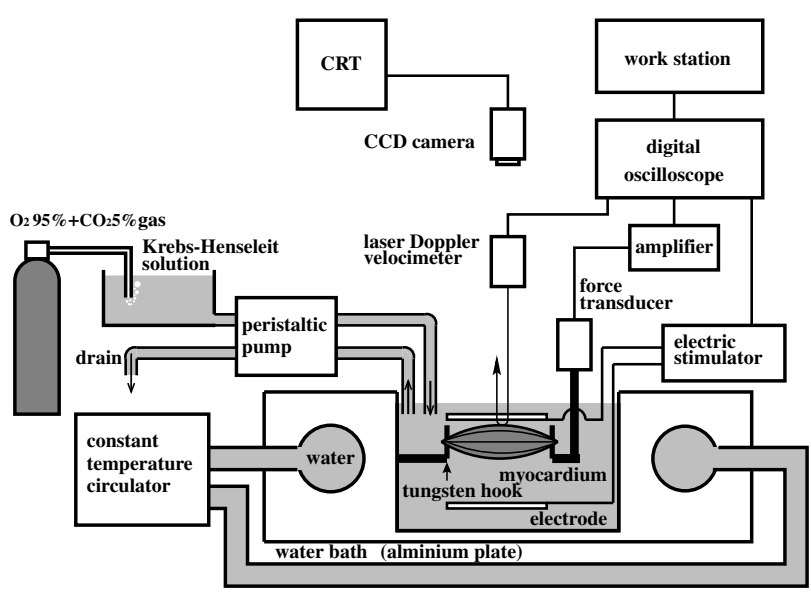

Fig. 1 Experimental setup for measuring the vibration of the papillary muscle extracted from the isolated rat heart, which is electrically stimulated during physiological solution perfusion.

shown in Fig. 3(b). The output $T(t)$ of the force transducer was obtained as shown in Fig. 3(d). These signals were simultaneously A/D converted at a sampling frequency of $50 \mathrm{kHz}$. They were then transfered to a workstation via a GPIB interface for further frequency analysis.

In order to detect the presence of contaminating noise component $n(t)$ in the measured velocity $v(t)$, the velocity component $n(t)$ at the arm of the tungsten hook was simultaneously measured as shown in Fig. 3(c). By integrating the velocity $v(t)$ of the myocardium in the workstation, its displacement $x(t)$ was estimated as shown in Fig. 3(e).

\section{IN VITRO EXPERIMENTAL RESULTS}

Each signal from Fig. 3(a) to Fig. 3(e) is divided into the respective duration periods and is overlaid in Fig. 2(a) to Fig. 2(e), where the horizontal time axis is expanded.

From Fig. 2(b), the velocity $v(t)$ of the myocardium included two types of components: one is the low frequency component which is synchronous with the electrically stimulated signal $e(t)$ in Fig. 2(a), and the other is the high frequency component which seems to be similar to the noise component $n(t)$ in the time domain of Fig. 2(c).

For detection of the change in power during one duration period in the frequency domain, the velocity signal $v(t)$ of each duration period in Fig. 3(b) was divided into 39 short-period signals $\{v(t ; i)\}(i=1,2, \ldots, 39)$ by shifting and multiplying the Hanning window with a length of $50 \mathrm{~ms}$ from the rising edge $(T=0)$ of the electrical stimulation $e(t)$ of each duration period, where half of the succeeding windows overlapped. Then, the Fourier transform was applied to each signal of $\{v(t ; i)\}$ $(i=1,2, \ldots, 39)$. The resultant power spectra $\{P(f ; i)\}$ up to $2 \mathrm{kHz}$ of the $i$ th short-period signals $\{v(t ; i)\}$ with the 


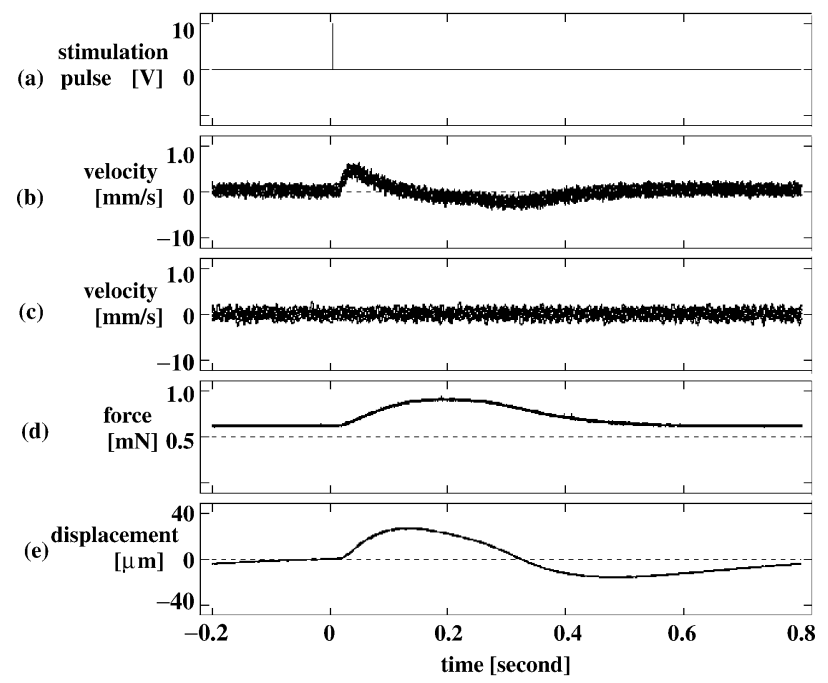

Fig. 2 Each signal from Fig. 3(a) to Fig. 3(e) is divided the respective duration periods and overlaid. For details, see the caption of Fig. 3.

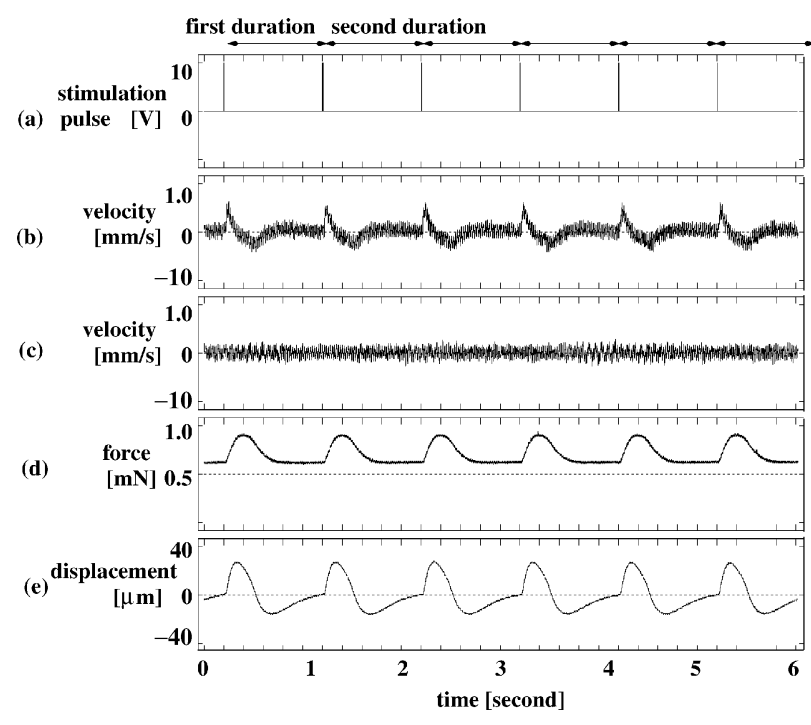

Fig. 3 For an uninjured heart muscle. (a) Rectangular pulse train $e(t)$ with a duration of $1 \mathrm{~ms}$ and a repetition frequency of $1 \mathrm{~Hz}$ for electrical stimulation of the myocardium. (b) Measured velocity component $v(t)$ generated in the direction normal to that of the myocardium measured by a laser Doppler velocimeter. (c) Output $T(t)$ of the force transducer. (d) Noise component $n(t)$ at the arm of the tungsten hook. (e) Displacement $x(t)$ of the myocardium estimated from $v(t)$ in Fig. (b).

same delay time from the rising edge were averaged for the 5 duration periods and are plotted in Fig. 4. In Fig. 4, the time origin $(T=0)$ corresponds to the rising edge of the electrical stimulation $e(t)$ of each duration period in Fig. 3(a). Each averaged power spectrum was normalized by the averaged power spectrum $\left\{P_{0}(f)\right\}$ at the period $200 \mathrm{~ms}$ in length just before the rising edge $(T=0)$. Thus, the value in Fig. 4 shows the change in the averaged power spectra

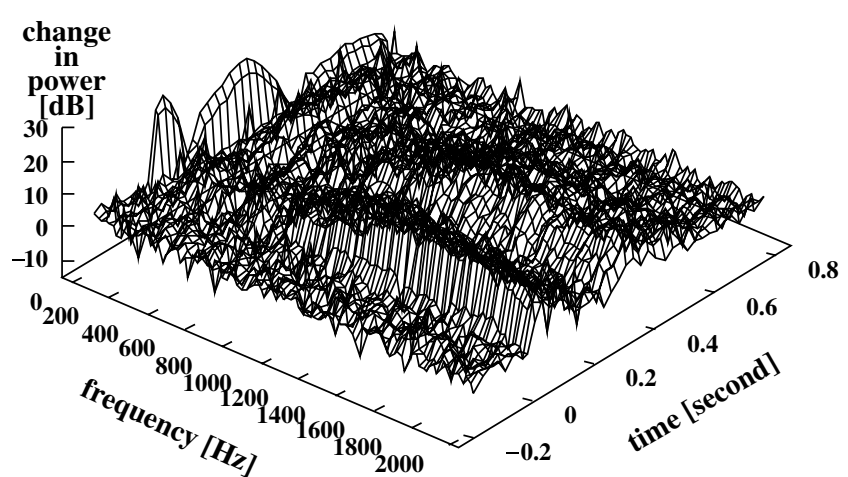

Fig. 4 For the velocity signal $v(t)$ in Fig. 3(b), the change in the averaged power spectra compared with that just before the contraction.

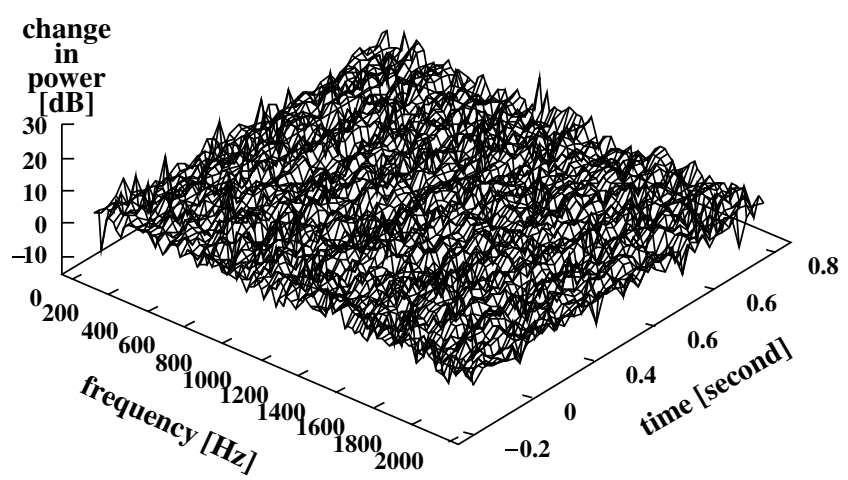

Fig. 5 For the noise signal $n(t)$ in Fig. 3(c), the change in the averaged power spectra compared with that just before the contraction.

compared with that just before the contraction.

The same procedure was applied to the noise signal $n(t)$ in Fig. 3(c) and the change in power spectrum is shown in Fig. 5. There did not seem to be any noteworthy change in power in Fig. 5. In Fig. 4, however, for the contraction period $(0-200 \mathrm{~ms})$ just after the electrical stimulation, there were large increases in power both in the low frequency component less than $25 \mathrm{~Hz}$ and in the wide frequency band from $200 \mathrm{~Hz}$ to $2 \mathrm{kHz}$. For the relaxation period (200$500 \mathrm{~ms}$ ) as well, there were large increases in power in both of these frequency bands as above. The low frequency component corresponds to the bending displacement of the muscle due to its contraction or the relaxation. For the higher frequency band, the power increased.

For the contraction period of $50 \mathrm{~ms}$ around the time ( $\left.T_{\mathrm{c}}\right) 75 \mathrm{~ms}$ after the rising edge $T_{0}$ and for the relaxation period with $50 \mathrm{~ms}$ around the time $\left(T_{\mathrm{r}}\right) 350 \mathrm{~ms}$ after $T_{0}$, the change in the averaged power spectrum compared with that just before the contraction in Fig. 4 are shown by red lines in Figs. 6(a) and 6(b), respectively. For the noise vibration $n(t)$ of the hook arm, the results in Fig. 5 are shown by blue lines in these figures. 

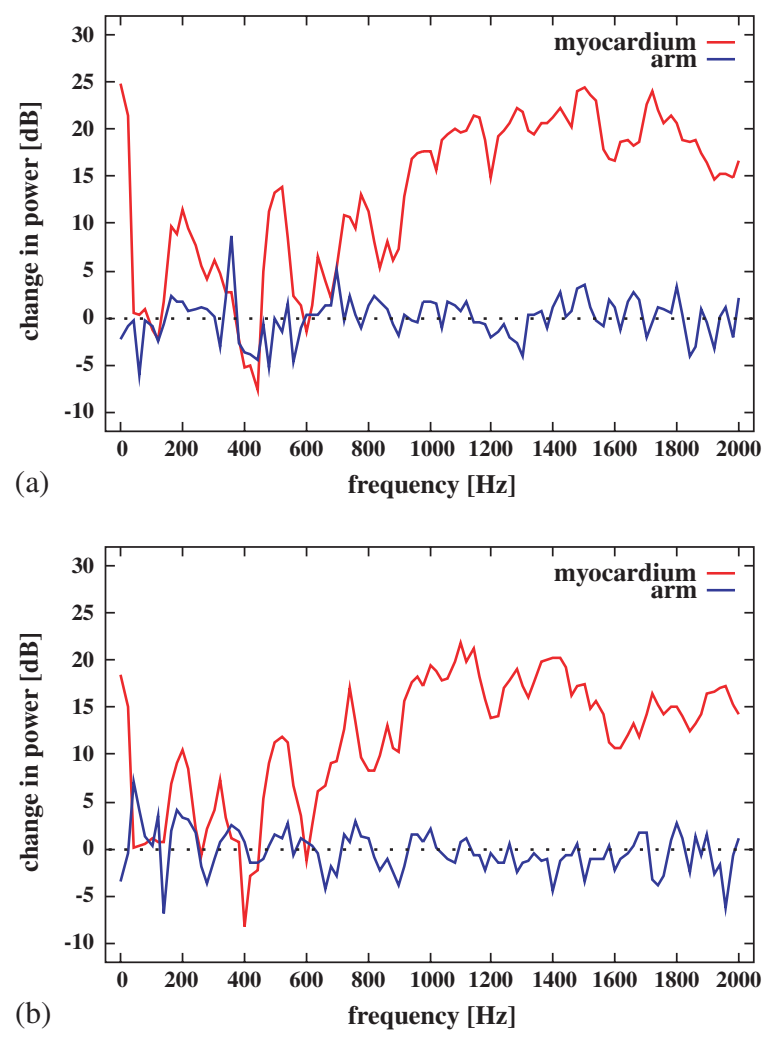

Fig. 6 For the contraction period with $50 \mathrm{~ms}$ around the time $\left(T_{\mathrm{c}}\right) 75 \mathrm{~ms}$ after the rising edge $T_{0}$, and for the relaxation period with $50 \mathrm{~ms}$ around the time $\left(T_{\mathrm{r}}\right)$ $350 \mathrm{~ms}$ after $T_{0}$, the change in the averaged power spectrum compared with that just before the contraction in Fig. 4 are shown by red lines in the upper (a) and lower (b) portion of the figure, respectively. For the noise vibration $n(t)$, the results in Fig. 5 are overlaid by blue lines.

As indicated by these blue lines, the noise vibration $n(t)$ did not show a noteworthy increase in power. For the vibration of the myocardium, however, the power increased by about $15-20 \mathrm{~dB}$ in the low frequency band less than $30 \mathrm{~Hz}$ and in the higher frequency band from $1 \mathrm{kHz}$ to $2 \mathrm{kHz}$. As indicated by these results, due to the contraction or the relaxation, telescopic vibrations with high frequency components in the frequency band from $1 \mathrm{kHz}$ to $2 \mathrm{kHz}$, which is other than the displacement, were certainly generated.

Next, in order to compare the characteristics of the above normal myocardium with those of an injured myocardium, for the same papillary muscle after measurement of the signals in Fig. 3, the perfusion with the KrebsHenseleit solution was completely stopped for 120 seconds. Then, the same measurement and analysis were applied to the injured myocardium.

The results are shown in Figs. 7-11. As for the measurement, the noise component $n(t)$ in Figs. 7(c) or 8(c) was large. However, this erroneous component was a low frequency component less than $40 \mathrm{~Hz}$ and was

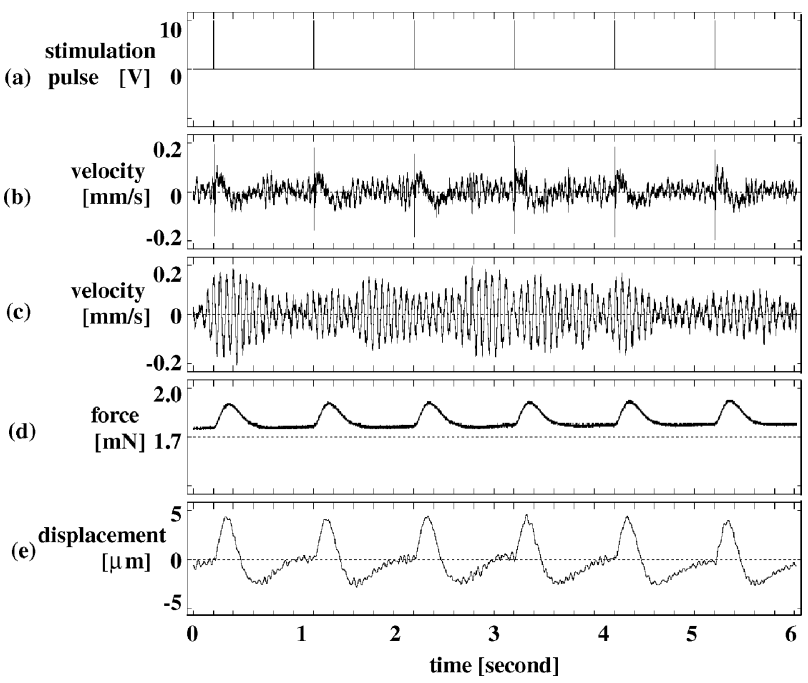

Fig. 7 For the injured heart muscle. For details, see the caption of Fig. 3.

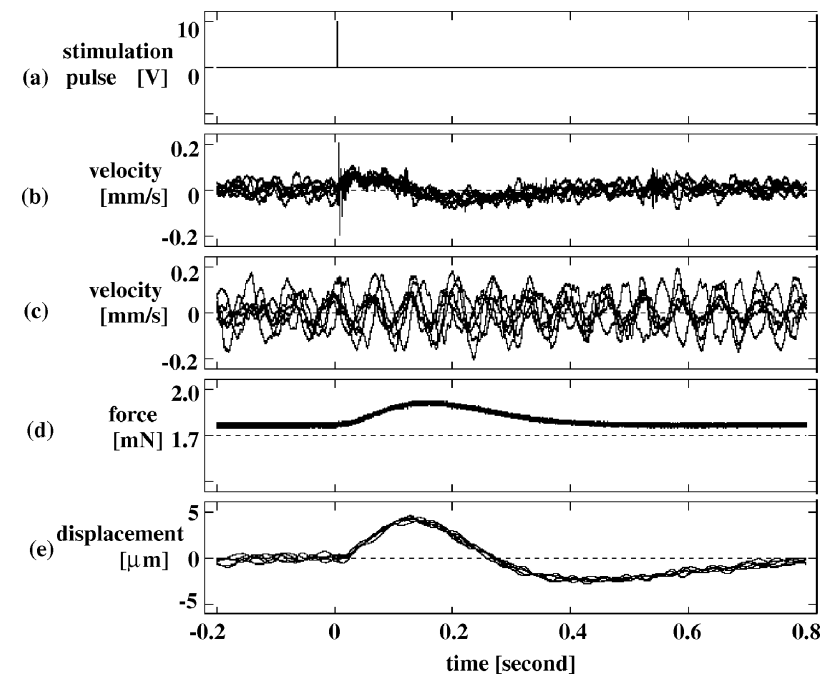

Fig. 8 For the injured myocardium, each signal from Fig. 7(a) to Fig. 7(e) is divided into the respective duration periods and overlaid. For details, see the caption of Fig. 2.

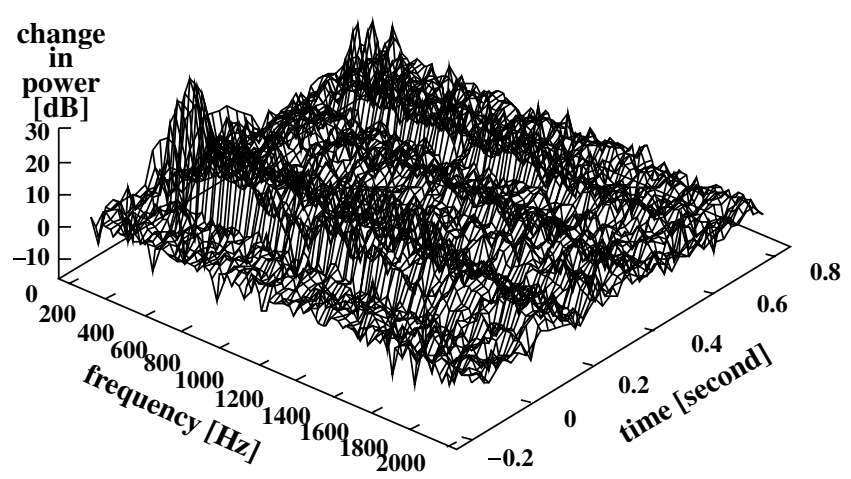

Fig. 9 For the velocity signal $v(t)$ in Fig. 7(b), the change in the averaged power spectra compared with that just before the contraction. 


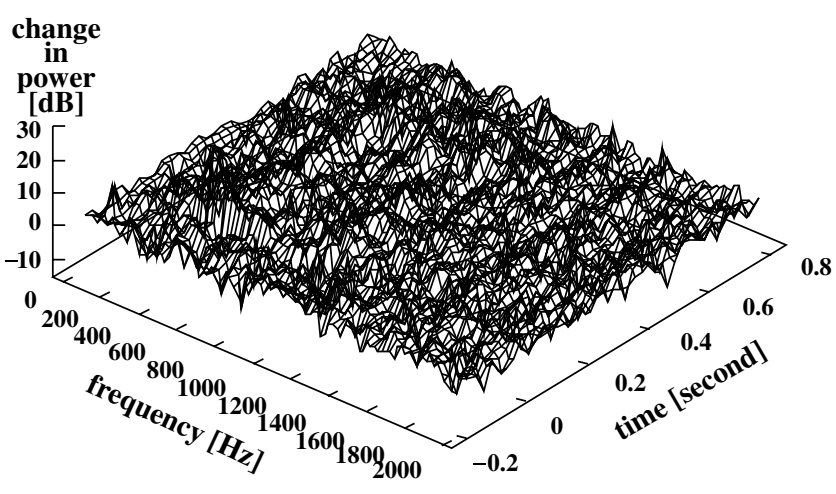

Fig. 10 For the noise signal $n(t)$ in Fig. 7(c), the change in the averaged power spectra compared with that just before the contraction.
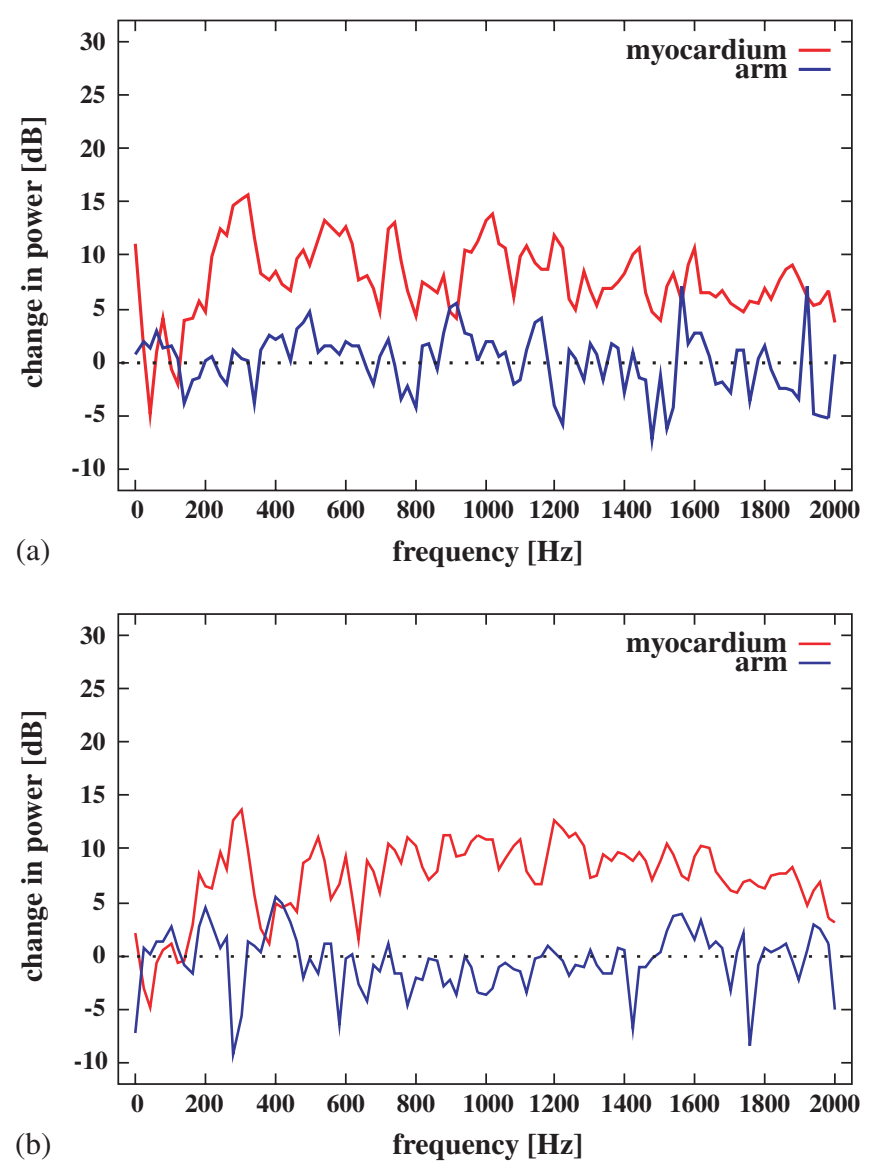

Fig. 11 For the injured myocardium, in the contraction period of $50 \mathrm{~ms}$ around the time $\left(T_{\mathrm{c}}\right) 75 \mathrm{~ms}$ after the rising edge $T_{0}$, and for the relaxation period of $50 \mathrm{~ms}$ around the time $\left(T_{\mathrm{r}}\right) 350 \mathrm{~ms}$ after $T_{0}$, the change in the averaged power spectrum compared with that just before the contraction in Fig. 9 are shown by red lines in the upper (a) and lower (b) portion of the figure, respectively. For the noise vibration $n(t)$, the results in Fig. 10 are overlaid by blue lines.

cancelled out by the change in the power spectrum component just before the contraction as shown in Fig. 10.

Also for the injured myocardium, a wide frequency band component was detected during the contraction and relaxation as shown in Figs. 9, 11(a), and 11(b). For the injured myocardium, however, the power increase was only about $10 \mathrm{~dB}$, which is not so large in comparison with those of the normal myocardium in Figs. 6(a) and 6(b), and the frequency band became lower at the point where the power increased.

\section{CONCLUSIONS}

In this paper, by electrically stimulating the extracted papillary muscle of rats, the vibration component in the direction normal to the myocardial fiber was measured by a laser Doppler velocimeter. Then, it was analyzed in the frequency domain. From the experimental results, the vibration during contraction and relaxation had a high frequency component from $1 \mathrm{kHz}$ to $2 \mathrm{kHz}$. For the injured myocardium, the power increase in the high frequency component during contraction or relaxation decreased. The experimental results support the fact that the high frequency component is included in the velocity signal measured for the first time for the human interventricular septum (IVS) by the phase tracking method using ultrasound [1]. Thus, the detection of the change in power has potential for diagnosis of the vulnerability of the regional heart muscle.

\section{REFERENCES}

[1] H. Kanai, M. Sato, Y. Koiwa and N. Chubachi, “Transcutaneous measurement and spectrum analysis of heart wall vibration," IEEE Trans. Ultrason. Ferroelectr. Freq. Control, 43, 791-810 (1996).

[2] H. Kanai, H. Hasegawa, N. Chubachi, Y. Koiwa and M. Tanaka, "Noninvasive evaluation of local myocardial thickening and its color-coded imaging," IEEE Trans. Ultrason. Ferroelectr. Freq. Control, 44, 752-768 (1997).

[3] H. Kanai, Y. Koiwa and J. Zhang, "Real-time measurements of local myocardium motion and arterial wall thickening," IEEE Trans. Ultrason. Ferroelectr. Freq. Control, 46, 1229-1241 (1999).

[4] H. Kanai, K. Sugimura, Y. Koiwa and Y. Tsukahara, "Accuracy evaluation in ultrasonic-based measurement of microscopic change in thickness," Electron. Lett., 35, 949-950 (1999).

[5] C. Orizio, R. Perini, B. Diemont, M. M. Figini and A. Veicsteinas, "Spectral analysis of muscular sound during isometric contraction of biceps brachii," J. Appl. Physiol., 68, 508-512 (1990).

[6] F. V. Brozovich and G. H. Pollack, "Muscle contraction generates discrete sound bursts," Biophys. J., 41, 35-40 (1983).

[7] D. T. Barry, "Acoustic signals from frog skeletal muscle," Biophys. J., 51, 769-773 (1987).

[8] D. T. Barry and N. M. Cole, "Muscle sounds are emitted at the resonant frequencies of skeletal muscle," IEEE Trans. Biomed. Eng., 37, 525-531 (1990).

[9] J. V. Frangioni, T. S. Kwan-Gett, L. E. Dobrunz and T. A. McMahon, "The mechanism of low-frequency sound production in muscle," Biophys. J., 51, 775-783 (1987). 


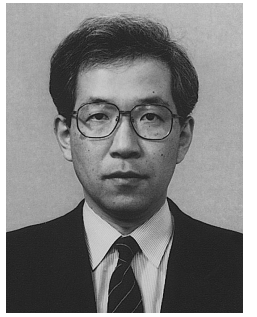

Hiroshi Kanai was born in Matsumoto, Japan, on November 29, 1958. He received a B.E. degree from Tohoku University, Sendai, Japan in 1981, and M.E. and the Ph. D. degrees, also from Tohoku University, in 1983 and in 1986, both in Electrical Engineering. From 1986 to 1990 he was with Processing, Tohoku University, as a research associate. From 1990 to 1992 he was a lecturer in the Department of Electrical Engineering, Faculty of Engineering, Tohoku University. From 1992 to 2001 he was an associate professor in the Department of Electrical Engineering, Faculty of Engineering, Tohoku University. Since 2001 he has been a professor in the Department of Electronic Engineering, Graduate School of Engineering, Tohoku University. His present interests are in ultrasonic measurement and digital signal processing for diagnosis of heart diseases and arteriosclerosis.

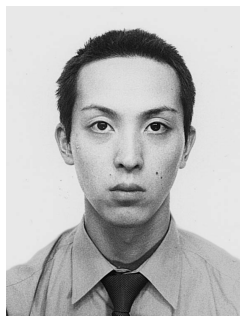

Shin-ichi Katsumata was born in Sendai, Japan, in 1977. He received a B.E. degree in Electrical Engineering from Tohoku University, Sendai, Japan in 2000. Since 2000, he has been a student of the Department of Electrical and Communication Engineering, Graduate School of Engineering, Tohoku University. His research interests are ultrasonic measurement and digital signal processing for myocardial

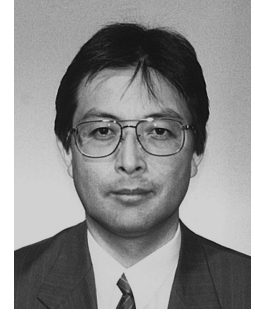

Hideyuki Honda was born in Matsushima, Japan, on June 9, 1953. He received a bachelor of Science Degree from Tohoku University, Sendai, Japan in 1977. He received MD degree and Ph.D. from Tohoku University School of Medicine, in 1985 and in 1992, respectively. From 1985 to 1987 , he was a resident fellow at Takeda general hospital, Aizuwakamatu, Japan. From 1992 to 1995 and 1995 to 1997, he was a Research Associate and Clinical Fellow at Tohoku University School of Medicine. From 1998 to 1999 , he was a Research Associate at the University of Illinois at Chicago. Since 1999, he has been an associate professor at Tohoku Bunka Gakuen University. His present interests are basic characteristics of muscles.

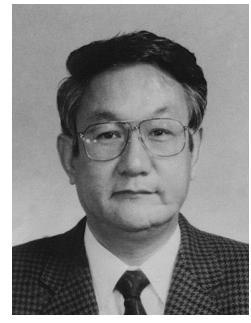

Yoshiro Koiwa was was born in Sendai, Japan, in 1944. He graduated from Tohoku University, Sendai, Japan, in 1969. He received the M.D. degree from Tohoku University in 1977. He is presently Associate Professor of Internal Medicine of Tohoku University. His main research interest is cardiovascular disease, especially cardiac function and heart failure. Dr. Koiwa is member of American Federation for Clinical Research, the Japanese Circulation Society, and the Japan Society of Medical Electronics and Biological Engineering. tissue characterization. 